\title{
Shifting Focus: From Hydration for Performance to Hydration for Health
}

\author{
Erica T. Perrier \\ Danone Research, Hydration and Health Department, Palaiseau, France
}

\author{
Keywords \\ Water · Hydration - Urine biomarkers - Vasopressin . \\ Copeptin $\cdot$ Kidney $\cdot$ Metabolism $\cdot$ Health $\cdot$ Disease
}

\begin{abstract}
Over the past 10 years, literature on hydration biomarkers has evolved considerably - from (de)hydration assessment towards a more global definition of biomarkers of hydration in daily life. This shift in thinking about hydration markers was largely driven by investigating the differences that existed between otherwise healthy individuals whose habitual, ad-libitum drinking habits differ, and by identifying physiological changes in low-volume drinkers who subsequently increase their water intake. Aside from obvious differences in urinary volume and concentration, a growing body of evidence is emerging that links differences in fluid intake with small, but biologically significant, differences in vasopressin (copeptin), glomerular filtration rate, and markers of metabolic dysfunction or disease. Taken together, these pieces of the puzzle begin to form a picture of how much water intake should be considered adequate for health, and represent a shifting focus from hydration for performance, toward hydration for health outcomes. This narrative review outlines the key areas of research in which the global hydration process - including water intake, urinary hydration markers, and vasopressin - has been associated with health outcomes, focusing on kidney and metabolic endpoints. It will also provide a commentary on how various hydration biomarkers may be used in hydration for health assessment. Finally, if adequate water intake can play a role
\end{abstract}

in maintaining health, how might we tell if we are drinking enough? Urine output is easily measured, and can take into account differences in daily physical activity, climate, dietary solute load, and other factors that influence daily water needs. Today, targets have been proposed for urine osmolality, specific gravity, and color that may be used by researchers, clinicians, and individuals as simple indicators of optimal hydration. However, there remain a large number of incomplete or unanswered research questions regarding the relationships between water intake, hydration, vasopressin, and health outcomes. Thus, this emerging field represents an excellent opportunity, particularly for young researchers, to develop relevant and novel lines of research.

(c) 2017 The Author(s)

Published by S. Karger AG, Basel

\section{Introduction}

Water has been described as the most essential nutrient: neglected, underappreciated, and under researched [1-4]. Early work on water intake, dehydration, and survival was conducted during the 1940s when Adolph published a series of experiments demonstrating that "life and efficiency in a hot climate depends upon the degree of dehydration, which is the overriding factor" $[5,6]$. However, the bulk of modern research into the effects of (de)hydration was not conducted until the 1970s through the 1990s, when a number of research groups began examining the acute effects of dehydration on physical performance. Conducted largely from the perspective of ath-

\section{KARGER}

E-Mail karger@karger.com www.karger.com/anm

\section{The Author(s) \\ Published by S. Karger AG, Basel}

This article is licensed under the Creative Commons AttributionNonCommercial-NoDerivatives 4.0 International License (CC BYUsage and distribution for commercial purposes as well as any distribution of modified material requires written permission.
Erica T. Perrier, $\mathrm{PhD}$, CSCS

Hydration and Health Department, Danone Research

Route Départementale 128

FR-91767 Palaiseau (France)

E-Mail erica.perrier@danone.com 
letic and military performance and safety, this body of work demonstrated conclusively that hypovolemia (a reduction in plasma volume) and hypertonicity (an increase in plasma osmolality) due to uncompensated sweat loss impact both cardiac output and thermal strain [7-9]. In order to minimize performance and safety concerns during exercise, authorities recommend limiting body water loss to no more than $2 \%$ of body mass $[7,10,11]$.

This body of work also had implications that reached beyond the realm of sports performance and safety. In establishing the Dietary Reference Values for water in 2004, the Institute of Medicine found insufficient evidence to set a water requirement as a means to treat or prevent a specific disease or condition [12]. Therefore, "hydration status, as assessed by plasma or serum osmolality, (was) the primary indicator" (pp. 144) was used to establish the Adequate Intake (AI) for water. The AI for total water was set as the median consumption reported in NHANES III, as serum osmolality was found to be unchanged across deciles of total water intake [13]. A normal hydration status, or hydration state (i.e., the maintenance of total body water content, represented by unchanged plasma osmolality) can be achieved with a broad range of total water intake volumes.

\section{To be, or Not to be, Dehydrated? That May No Longer be the Question}

The concept of hydration as a state, representing the amount of water in the body at a given time, is highly relevant for short-term performance and safety, particularly during physical activity in hot environments or in the absence of free water access. The hydration state is also of critical concern in vulnerable populations, including the elderly, who have an increased prevalence of plasma hypertonicity [14]. Several studies suggest that dehydration is associated with increased mortality rates among hospitalized older adults $[15,16]$. Moreover, dehydration may precipitate emergency hospitalization or increase the risk of repeated hospitalization $[15,17,18]$, and some evidence suggests that in the elderly, dehydration may contribute to constipation, impaired cognitive function, falling, orthostatic hypotension, salivary dysfunction, poor control of hyperglycemia in diabetes, or hyperthermia; and that water supplementation may alleviate some of these conditions [18-21]. In considering this evidence, it is important to remember that older adults represent a vulnerable population with specific hydration challenges, including reduced thirst, lower lean body mass (and thus

Shifting Focus: From Hydration for Performance to Hydration for Health lower body water), and a reduced capacity to concentrate urine. In contrast, there is little to no evidence that any significant proportion of the general, healthy population is chronically dehydrated.

Thus, if we restrict our definition of hydration to that of a state, there is little evidence for broadly generalizable health benefits. Yet, over the past decade, the hydration research landscape has shifted from studying hydration as a state to investigating the processes and mechanisms through which the hydration state is maintained. While individuals who consume low fluid volumes ad-libitum ("low drinkers") maintain a normal plasma osmolality, other physiological markers are significantly different between low-volume and high-volume drinkers. Low drinkers have significantly lower 24 -h urine volume, higher urine concentration (osmolality, specific gravity, and color) [22-27] and, in some studies, demonstrate higher plasma vasopressin (AVP) or even higher cortisol [2729], compared to their high-drinking counterparts. Although the values for urinary and blood markers in low drinkers remain within normal limits, these differences suggest that chronic low drinking triggers a sustained antidiuretic response to conserve body water using the normal, renal concentrating mechanism. Shifting the paradigm toward redefining hydration as a dynamic, ongoing process to maintain water balance allows for distinctions between low total water intake, and low urinary output; from high water intake, and high urinary output [30]. Recent studies linking low water intake, low urine output, or high AVP (or its surrogate, copeptin) to various health outcomes or risk factors [31-39] suggest that maintaining the hydration state in the face of habitual low intake has a cost. The entire process of hydration (from what goes in, to what comes out, to what happens internally to maintain body water homeostasis) may be more important than the hydration state when considering implications for kidney or cardiometabolic health (Fig. 1) [40]. Thus, the time is ripe for a fundamental shift in the research questions linked to hydration: what are the metabolic or health costs of maintaining the hydration state when drinking is low?

\section{What Goes In: Associations between Total Fluid Intake, Plain Water Intake, and Kidney and Metabolic Health}

Multiple associations have been reported between fluid intake and kidney and metabolic health outcomes, including chronic kidney disease (CKD), recurrence of kidney 
Fig. 1. Connecting the dots: links between fluid intake, hydration biomarkers, vasopressin, and health outcomes. Adapted from Guelinckx et al. [40].

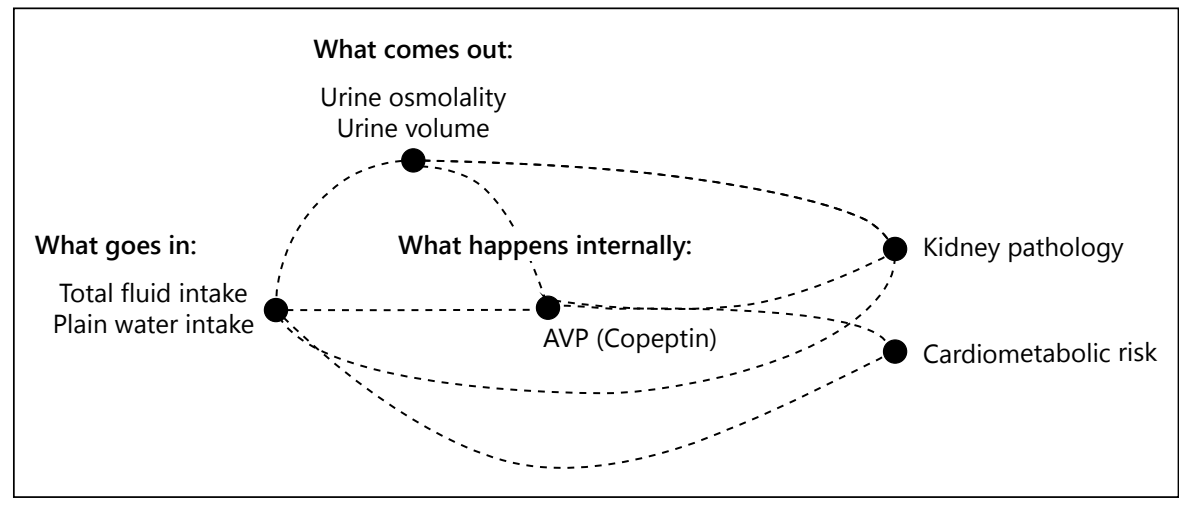

stones, and some metabolic markers of hyperglycemia. In an Australian cohort, Strippoli et al. [41] found that individuals with the highest quintile of fluid intake had significantly reduced risk of CKD. In a follow-up publication, this same research group further reported that if one excludes plain water from the analysis [42], there was no longer an association with kidney function or with cardiovascular or all-cause mortality, suggesting that plain water intake may play a determining role. This is further supported by an analysis of NHANES data by Sontrop et al. [32], who found CKD prevalence to be the highest among those with the lowest total water intake. When intake was subdivided into plain water versus other beverages, the association was found to hold only for plain water, further suggesting that water, in particular, may play a protective role in kidney disease. Higher total fluid intake has also been associated with lower relative risk for kidney stones in the Nurses' Health Study II [43], and an intervention to increase water intake reduced recurrence of kidney stones in a randomized, controlled trial [39]. In terms of metabolic disease endpoints, low water intake has been associated with new-onset hyperglycemia in a prospective French cohort with a 9-year follow-up [31]. More recently, Carroll et al. [38] also reported an association between higher plain water intake and lower HbAlc in men, but not in women, participating in the UK National Diet and Health Survey. Their substitution analysis revealed that the effect of plain water was not due to substituting water for other beverages; rather, it was the addition of plain water that appeared to be the pertinent factor. Overall, a key challenge is to design and carry out intervention studies in order to demonstrate that fluid intake is causally related to kidney and metabolic health endpoints. Nevertheless, we appear to be approaching a critical mass of associative evidence for the role of fluid intake, and specifically plain water intake, in kidney and cardiometabolic health.

\section{What Comes Out: Relationships between Urinary Output and Kidney Health}

High urine volume (and low urine concentration) is the logical end result of a high fluid intake. It is not surprising, then, that the associations seen between fluid intake and kidney health endpoints are also reported using urinary output as a predictor. Clark et al. [33] found that maintaining a high versus low 24 -h urine volume reduced the rate of estimated glomerular filtration rate (GFR) decline in a prospective cohort with a 5.7-year follow-up. In another short-term intervention study, increasing urine volume and lowering urine concentration by drinking more water was also shown to reduce the Tiselius Crystallization Risk Index in 24-h urine samples, a risk factor for recurrent kidney stones [44]. The European Urology Association also recommends that a low urine concentration be maintained in order to prevent stone recurrence [45]. For stone disease, it is likely that reducing urine concentration simply decreases the likelihood for supersaturation and thus crystal formation. For CKD, however, the explanation for why increased urine output may be protective is more complex. Clark et al. [33] and Sontrop et al. [32] offer several hypotheses: the kidney requires a minimum "obligatory" urine volume to remove waste, and it is possible that the kidneys function more efficiently with a more abundant supply of water. It is also possible that the production of more concentrated urine incurs a greater metabolic demand on the kidney [33], or that higher urine concentration may, over time, contribute to glomerular hyperfiltration and albuminuria [32]. One significant challenge in assessing the relation between urinary output and health endpoints is that in a majority of cohorts, if urine is collected at all, it is a single (random) or morning (fasting) sample, which may or may not represent 24-h urine. The existing literature, however, con- 
sistently supports a benefit of increased fluid or plain water intake. One possible solution to the challenge of collecting 24-h urine samples in a large cohort is to instead evaluate plasma copeptin as a surrogate to vasopressin, as an indicator of the activity of the urine concentrating or diluting mechanism, and as an indicator of hydration.

\section{What Happens Internally: Relationships between Copeptin (Vasopressin), Kidney, and Metabolic Health}

It is difficult to measure the levels of vasopressin, the primary hormone responsible for regulating water reabsorption in the kidney, under normal circumstances, as its concentration in the blood is quite low (25th-75th percentile in one of our research group's prior studies: 1.1$3.4 \mathrm{pmol} \cdot \mathrm{L}^{-1}[27]$ ) and its half-life is short (i.e., $10-20 \mathrm{~min}$ in plasma). Vasopressin is secreted in a 1:1 ratio along with copeptin and neurophysin II, which together form the pre-pro-vasopressin precursor. The recent development of a sensitive copeptin assay kit for clinical use [46] has popularized the measurement of copeptin in clinical studies, where it is considered to be a surrogate measure for vasopressin. Over the past several years, plasma copeptin has also been measured in some cohort studies, allowing for associations to be made with health outcomes. Copeptin has been independently associated with diabetes, metabolic syndrome, abdominal obesity, metabolic heart disease, and death [34-37], as well as with GFR decline and progression toward CKD [47, 48]. One limitation is that widespread copeptin measurement is relatively new, and thus the number of large cohorts is limited. A majority of the available results were obtained from analyses performed on the Malmö Diet and Cancer Study (Sweden) and D.E.S.I.R. (France) cohorts $[47,48]$. One major strong point of the analyses, however, is that copeptin remained an independent risk factor after controlling for covariates most often associated with kidney and metabolic disease. The inclusion of copeptin in future cohorts, or analysis of stored blood samples in existing cohorts, would facilitate the confirmation of these findings.

\section{Assessing Hydration from a Health Perspective}

Given the evidence described above, a strong case exists for pursuing further research into the links between the global hydration process (including water intake, urinary output, and copeptin concentration) and health.
Selecting the appropriate biomarkers to assess the global hydration process, and measuring them under the right conditions, is thus fundamental to advancing the research on hydration and health. This is especially important when considering that in different experimental conditions, the same biomarker may be an indicator of the hydration state and of the global hydration process to maintain total body water or both. Consider the following 2 studies on similar populations but with vastly different protocols: Munoz et al. [49] subjected young, healthy volunteers to both passive (sedentary) and active (exercise) dehydration in the heat; serum osmolality, body mass loss, and urinary hydration biomarkers were tracked throughout the progressive body water loss. In a separate study, Perrier et al. [27] measured plasma and urinary hydration biomarkers in young, healthy adults with habitually different fluid intakes (low: $0.74 \mathrm{~L} /$ day; high: $2.70 \mathrm{~L} /$ day) over 4 consecutive days, with no dehydration intervention. Figure 2 illustrates plasma (or serum) osmolality $\left(\mathrm{P}_{\mathrm{Osm}}\right)$, urine osmolality $\left(\mathrm{U}_{\mathrm{Osm}}\right)$, and urine specific gravity $\left(\mathrm{U}_{\mathrm{SG}}\right)$ at baseline vs. $1 \%$ body mass loss for the dehydration study [49], and for high vs. low drinkers for the habitual fluid intake study [27]. Only during the active dehydration condition, did $\mathrm{P}_{\mathrm{Osm}}$ significantly rise at $1 \%$ body mass loss. Conversely, a significant change in urine concentration ( $\mathrm{U}_{\mathrm{Osm}}$ and $\mathrm{U}_{\mathrm{SG}}$ ) was detected at $1 \%$ body mass loss only for the passive dehydration condition, and for high vs. low drinkers. High urine concentration may thus indicate mild, gradual dehydration, or chronic low fluid intake, depending upon the context, whereas a change in plasma osmolality is a sensitive measure of mild to moderate acute dehydration but does not appear to differ as a function of fluid intake or during mild passive body water loss.

\section{Urine Color as a Hydration Marker with Real-Life Utility}

While $U_{\text {Osm }}$ and $U_{S G}$ may be used in research or clinical practice as hydration markers, both measures require some degree of specialized equipment and technical expertise, and are thus less well-suited for day-to-day hydration monitoring. Individualized hydration tracking via a portable densitometer has been shown to be feasible for daily hydration monitoring and the secondary prevention of urinary tract infection [50], and maintaining a $\mathrm{U}_{\mathrm{SG}}$ below 1.010 is recommended in the secondary prevention of kidney stone disease [45]. While patients may be motivated to track hydration via $U_{S G}$ to prevent recurrence, a simpler 


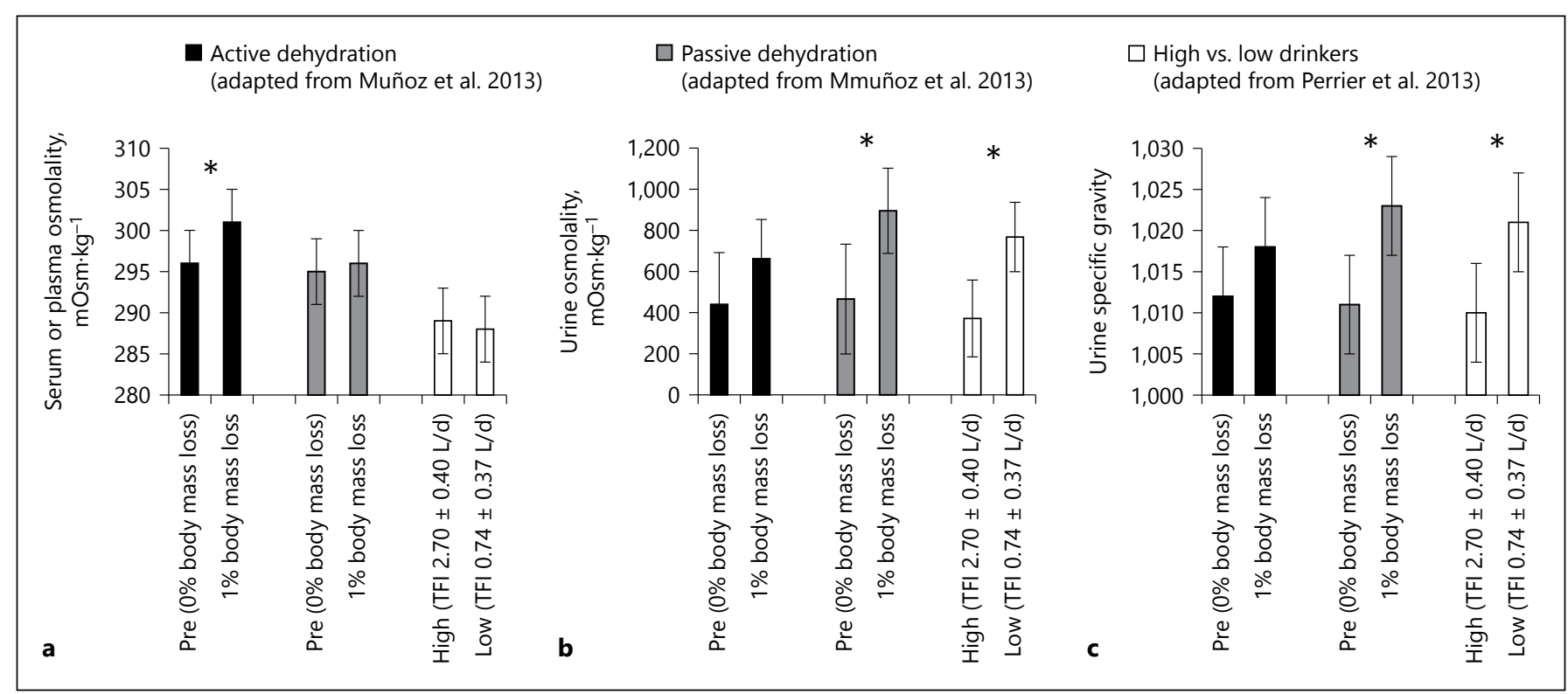

Fig. 2. Plasma (or serum) osmolality (a), urine osmolality (b), and urine specific gravity (c) under 3 different experimental conditions: at baseline and $1 \%$ body mass loss during active dehydration; at baseline and $1 \%$ body mass loss during passive dehydration; and in response to differences in habitual fluid intake. Serum osmolal- ity increased in response to mild, active dehydration, whereas urinary biomarkers were responsive to passive dehydration and differences of fluid intake. ${ }^{*} p$ value $<0.05$. TFI, total fluid intake (all sources); high, high-volume drinkers; low, low-volume drinkers. method to increase awareness of drinking habits for the general population is to simply monitor urine color $\left(\mathrm{U}_{\mathrm{Col}}\right)$. Using an 8-point color scale originally developed by Armstrong et al. [51, 52] for dehydration assessment, the utility of $\mathrm{U}_{\mathrm{Col}}$ to accurately detect elevated $\mathrm{U}_{\mathrm{Osm}}$ has been demonstrated in healthy adults [53], children [54], and in pregnant and breastfeeding women [55]. Periodically monitoring $\mathrm{U}_{\mathrm{Col}}$ and increasing water intake in response to more concentrated urine (shade 4 or higher on the 8 -point scale) is likely a sufficient level of hydration monitoring for most individuals in the general population.

\section{Twenty-Four Hour, First Morning, and Single Urine Sampling for Tracking Hydration Process}

Because fluid intake occurs periodically throughout the day, and not on a set schedule [56], and because there exists circadian variation in vasopressin [57] that, independently of fluid intake, influences urinary excretion [24], the hydration process is best assessed through the collection of a 24-h urine sample. Twenty-four-hour urine concentration also tracks day-to-day changes of daily fluid intake $[24,28,29]$, and thus it can be used as a clinical measure during fluid intake studies as an outcome or as an indicator of subject compliance with drinking instructions. However, 24-h collections are cumbersome, often difficult for subjects, and impractical for any research situation other than short-term clinical trials. This poses a particular challenge in linking the hydration process to long-term health outcomes, as first evidence for associations is often obtained from large cohort studies, which cannot ensure 24 -h collections on large sample sizes in free-living conditions.

Larger studies and cohorts such as NHANES in the United States, and the HELENA study in Europe, typically instead obtain single urine samples, which may represent either the first morning collection or a random sample obtained during the day [58]. However, first morning samples tend to overestimate 24 -h urine concentration [22], even under situations of controlled fluid intake [24], since AVP levels peak overnight and sleeping effectively represents an overnight fluid restriction. Further, the difference between first morning and 24-h urine concentration may be dependent upon fluid intake volume. For low drinkers, who are essentially in an antidiuretic state all day, we previously observed that first morning $\mathrm{U}_{\text {Osm }}$ may not at all be different than the 24-h value (794 vs. $767 \mathrm{mOsm} \cdot \mathrm{kg}^{-1}$, respectively); whereas for high drinkers, the difference between the first morning and $24-\mathrm{h}$ 
sample may be quite large (590 vs. $371 \mathrm{mOsm} \cdot \mathrm{kg}^{-1}$ ) [27]. Thus, even the use of a correction factor to adjust first morning urine values may prove to be complex.

Another approach involves determining a window during which a single urine sample would reasonably approximate $24-\mathrm{h} \mathrm{U}_{\mathrm{Osm}}$. To date, and to our knowledge, only one study has evaluated the equivalence of single to 24-h $U_{\text {Osm }}$ in healthy, free-living young adults, with the aim of establishing a collection window that may provide guidance as to the best time for single urine collection in research and clinical practice. The results suggest that $\mathrm{U}_{\text {Osm }}$ measured on urine samples obtained in mid-afternoon to early evening (14:00-20:00) was equivalent (defined as falling within $100 \mathrm{mOsm} \cdot \mathrm{kg}^{-1}$ ) to the 24 -h value, while samples taken earlier in the day tended to overestimate 24-h $\mathrm{U}_{\mathrm{Osm}}$ [59]. One caveat to this conclusion, however, is that this study was performed in European subjects with Western dietary practices and meal times. It is possible that substantial deviations from this eating and drinking pattern, or night and shift work, may alter the window during which equivalence can be expected.

\section{Reference Values and Cut-Offs: What is the Target for Optimal Hydration?}

A key question in hydration assessment involves determining the thresholds or cut-off values that represent adequate hydration for health outcomes. A commonly referenced cut-off for the upper limit of euhydration is a $\mathrm{U}_{\text {Osm }}$ of $800 \mathrm{mOsm} \cdot \mathrm{kg}^{-1}$, which has been used in studies of hydration in both children [60-63] and adults [64, 65]. The principle behind the $800 \mathrm{mOsm} \cdot \mathrm{kg}^{-1}$ cut-off has roots in early work by Katz et al. [66], who studied the urine-concentrating capacity of the kidney in hot conditions, and determined that nearly all participants could concentrate their urine up to at least $800 \mathrm{mOsm} \cdot \mathrm{kg}^{-1}$. This concept of the "minimum, maximum urine concentrating capacity" was also later explored by Manz and Wentz [67], who suggested that an upper limit for euhydration could be calculated as the mean maximum $\mathrm{U}_{\mathrm{Osm}}$, minus 2 SDs, as way to ensure euhydration for nearly all members of a given population. However, with the emerging distinction between the hydration state and hydration process [30], and with recent associations between low water intake, low urinary output, higher plasma copeptin, and kidney and metabolic outcomes [31-37], a lower target for 24-h urine concentration may be warranted.

Our research group has proposed a target for 24-h $\mathrm{U}_{\text {Osm }}$ of $500 \mathrm{mOsm} \cdot \mathrm{kg}^{-1}$ [68], which represents a $\mathrm{U}_{\text {Osm }}$

Shifting Focus: From Hydration for

Performance to Hydration for Health that is consistent with daily intake sufficient to replace all water losses, ensure a sufficient urinary output to reduce the risk of kidney-related pathologies, and which may reduce circulating plasma vasopressin (copeptin). Given the growing evidence linking water intake, urine output, and copeptin to not only the kidney but also to cardiometabolic health, we argue that this value represents a reasonable target for 24-h hydration in the general population, and may contribute to reducing kidney and cardiometabolic risk over time. In order to expand the utility of this cut-off beyond clinical research, we also calculated the optimal thresholds for $\mathrm{U}_{\mathrm{SG}}(\geq 1.013)$ and participant-assessed $\mathrm{U}_{\mathrm{Col}}(\geq 4)$ to detect $\mathrm{U}_{\mathrm{Osm}}>500$ $\mathrm{mOsm} \cdot \mathrm{kg}^{-1}$ [53], thus opening the door for clinicians, health care practitioners, and individuals to monitor and adjust hydration behaviors accordingly.

These values represent a first, but not definitive, step toward refining and obtaining consensus regarding a target urine concentration for being well hydrated. In a recent publication, Cheuvront and Kenefick [69] acknowledge that there may be room for a "gray area between dehydration and euhydration," which may be of interest in longterm health. Hydration, then, may be best viewed as a continuum between dehydration (fluid losses exceed fluid gains), the lower limit of euhydration (fluid losses are compensated by fluid gains; however, the kidneys conserve body water in order to avoid excess losses, resulting in low urine volume, high urine concentration, and increased circulating vasopressin), and being well hydrated (fluid losses are compensated by fluid gains, with a water reserve that allows for ample urine output, at a lower concentration, accompanied by lower circulating vasopressin). It is the latter, that is, being well hydrated, that may be the most important to long-term health, but Cheuvront and Kenefick [69] also rightly point out that the existing research is insufficient. Today, while there is some good evidence for increased water intake for renal health, the evidence linking being well hydrated to other health benefits, including cardiometabolic health, is more limited, is primarily based upon associations observed in large cohorts, and is centered around copeptin (vasopressin) as an independent predictor of health outcomes. While vasopressin is the central node in a working model linking water intake, hydration, and health (Fig. 1), more evidence is required to demonstrate a causal relationship between vasopressin and metabolism, and to demonstrate that increasing water intake can reduce circulating vasopressin and improve risk markers for disease.

After Cheuvront and Kenefick's paper was published in early 2016 [69], another study was published, which offers a first suggestion of a causal relationship between va- 
sopressin and metabolism. Roussel et al. [70] investigated associations between baseline plasma copeptin and various glycemic and insulinemic risk markers for metabolic disease in a large French cohort with a 9-year follow-up. To address the question of causality between vasopressin and disease, they looked at several single nucleotide polymorphisms in the AVP locus and assessed the relationship between AVP gene variants, copeptin, and clinical outcomes. They found that baseline plasma copeptin concentration was associated with lower insulin sensitivity as well as increased incidence of impaired fasting glucose or Type 2 diabetes mellitus (IFG/T2DM) during follow-up. Moreover, they also reported that in men, genotypes associated with higher circulating copeptin also showed a higher cumulative incidence of IFG/T2DM. This pattern of Mendelian randomization, though not a conclusive demonstration of causality, represents the first suggestion of a causal relationship between vasopressin and IFG/T2DM.

Moreover, there also exists first evidence that normal, healthy adults can lower circulating plasma copeptin over the medium-term by increasing water intake. In a stratified sample of young French adults with habitual low, medium, and high fluid intake, we found that baseline plasma copeptin was associated with $24-\mathrm{h} \mathrm{U}_{\text {Osm }}$ (unpublished observations); then, in the low- and medium-drinkers, a 6-week water intake intervention resulted in a significant decrease of circulating plasma copeptin. While this was a secondary analysis, and although it contained certain experimental limitations, this study demonstrated that plasma copeptin can be modified through increased water intake, even in young, healthy individuals with normal baseline copeptin levels.

\section{Perspectives for Future Research}

The existing literature allows a working model of fluid intake, hydration, and health to be put into place. However, the level of evidence for each link in the model is uneven, and many research questions remain insufficiently answered. With regard to plasma copeptin, fluid intake, and hydration, we must quantify the relationships between urinary hydration biomarkers and copeptin, and quantify changes in copeptin in response to increased water intake in a broader range of subjects. The proposed threshold of $500 \mathrm{mOsm} \cdot \mathrm{kg}^{-1} \mathrm{U}_{\text {Osm }}$ requires more research to support, build upon, or update this target. With respect to copeptin and disease, thresholds for copeptin that are predictive of disease in men and women are needed; then, it will be necessary to demonstrate that increasing water intake to lower copeptin also impacts risk markers for disease. Finally, there is a paucity of research regarding ways that hydration and copeptin may impact specific segments of the population. In pregnant women, limited research suggests that plasma copeptin as early as the first trimester may be predictive of preeclampsia [71, 72]; in children, research regarding water intake, hydration, and vasopressin is virtually nonexistent outside of enuresis. Today, the field is wide open and, for young researchers in particular, represents a huge opportunity for novel research.

\section{Disclosure Statement}

E.T.P. is a full-time employee of Danone Research.

\section{References}

1 Rush EC: Water: neglected, unappreciated and under researched. Eur J Clin Nutr 2013; 67:492-495

2 Jequier E, Constant F: Water as an essential nutrient: the physiological basis of hydration. Eur J Clin Nutr 2010;64:115-123.

3 Kavouras SA, Anastasiou CA: Water physiology: essentiality, metabolism, and health implications. Nutr Today 2010;45:S27-S32.

4 Manz F, Wentz A, Sichert-Hellert W: The most essential nutrient: defining the adequate intake of water. J Pediatr 2002;141: 587-592.

5 Adolph EF: Physiology of Man in the Desert. New York, Interscience Publishers, Inc., 1947.

$6 \mathrm{HH}$ : Physiology of man in the desert (book review). Br J Ind Med 1948;5:214.
7 Cheuvront SN, Carter R 3rd, Sawka MN: Fluid balance and endurance exercise performance. Curr Sports Med Rep 2003;2:202208.

8 Sawka MN, Montain SJ, Latzka WA: Hydration effects on thermoregulation and performance in the heat. Comp Biochem Physiol A Mol Integr Physiol 2001;128:679-690.

9 Sawka MN, Young AJ, Francesconi RP, Muza SR, Pandolf KB: Thermoregulatory and blood responses during exercise at graded hypohydration levels. J Appl Physiol (1985) 1985;59: 1394-1401.

10 Casa DJ, Armstrong LE, Hillman SK, Montain SJ, Reiff RV, Rich BS, Roberts WO, Stone JA: National Athletic Trainers' Association position statement: fluid replacement for athletes. J Athl Train 2000;35:212-224.
11 American College of Sports Medicine, Sawka MN, Burke LM, Eichner ER, Maughan RJ, Montain SJ, Stachenfeld NS: American College of Sports Medicine position stand. Exercise and fluid replacement. Med Sci Sports Exerc 2007;39:377-390.

12 Institute of Medicine (IOM): Dietary Reference Intakes for Water, Potassium, Sodium, Chloride, and Sulfate. Washington, National Academies Press, 2004.

13 US Department of Health and Human Services NCfHS: Third National Health and Nutrition Examination Survey(NHANES III), Appendix Table G-1, 1988-1994.

14 Stookey JD: High prevalence of plasma hypertonicity among community-dwelling older adults: results from NHANES III. J Am Diet Assoc 2005;105:1231-1239. 
15 Mentes J: Oral hydration in older adults: greater awareness is needed in preventing, recognizing, and treating dehydration. Am J Nurs 2006;106:40-49; quiz 50.

16 Warren JL, Bacon WE, Harris T, McBean AM, Foley DJ, Phillips C: The burden and outcomes associated with dehydration among US elderly, 1991. Am J Public Health 1994;84: 1265-1269.

17 Begum MN, Johnson CS: A review of the literature on dehydration in the institutionalized elderly. E Spen Eur E J Clin Nutr Metab 2010;5:e47-e53.

18 Robinson SB, Rosher RB: Can a beverage cart help improve hydration? Geriatr Nurs 2002; 23:208-211.

19 Suhr JA, Hall J, Patterson SM, Niinisto RT: The relation of hydration status to cognitive performance in healthy older adults. Int J Psychophysiol 2004;53:121-125.

20 Manz F: Hydration and disease. J Am Coll Nutr 2007;26(suppl):535S-541S.

21 Anti M, Pignataro G, Armuzzi A, Valenti A, Iascone E, Marmo R, Lamazza A, Pretaroli AR, Pace V, Leo P, Castelli A, Gasbarrini G: Water supplementation enhances the effect of high-fiber diet on stool frequency and laxative consumption in adult patients with functional constipation. Hepatogastroenterology 1998;45:727-732.

22 Armstrong LE, Pumerantz AC, Fiala KA, Roti MW, Kavouras SA, Casa DJ, Maresh CM: Human hydration indices: acute and longitudinal reference values. Int J Sport Nutr Exerc Metab 2010;20:145-153.

23 Armstrong LE, Johnson EC, Munoz CX, Swokla B, Le Bellego L, Jimenez L, Casa DJ, Maresh CM: Hydration biomarkers and dietary fluid consumption of women. J Acad Nutr Diet 2012;112:1056-1061.

24 Perrier E, Demazieres A, Girard N, Pross N, Osbild D, Metzger D, Guelinckx I, Klein A: Circadian variation and responsiveness of hydration biomarkers to changes in daily water intake. Eur J Appl Physiol 2013;113:2143-2151.

25 Armstrong LE, Johnson EC, Munoz CX, Le Bellego L, Klein A, McKenzie AL, Casa DJ, Maresh CM: Evaluation of Uosm: Posm ratio as a hydration biomarker in free-living, healthy young women. Eur J Clin Nutr 2013; 67:934-938.

26 Perrier E, Rondeau P, Poupin M, Le Bellego L, Armstrong LE, Lang F, Stookey J, Tack I, Vergne S, Klein A: Relation between urinary hydration biomarkers and total fluid intake in healthy adults. Eur J Clin Nutr 2013;67:939943.

27 Perrier E, Vergne S, Klein A, Poupin M, Rondeau P, Le Bellego L, Armstrong LE, Lang F, Stookey J, Tack I: Hydration biomarkers in free-living adults with different levels of habitual fluid consumption. Br J Nutr 2013;109: 1678-1687.

28 Johnson EC, Armstrong LE: Switching habitual small and large volume drinkers: outcomes and lessons learned. Nutr Today 2013; $48: S 36-S 39$
29 Johnson EC, Muñoz CX, Jimenez L, Le Bellego L, Kupchak BR, Kraemer WJ, Casa DJ, Maresh CM, Armstrong LE: Hormonal and thirst modulated maintenance of fluid balance in young women with different levels of habitual fluid consumption. Nutrients 2016; 8:pii:E302.

30 Perrier ET, Armstrong LE, Daudon M, Kavouras S, Lafontan M, Lang F, Peronnet F, Stookey JD, Tack I, Klein A: From state to process: defining hydration. Obes Facts 2014 7(suppl 2):6-12.

31 Roussel R, Fezeu L, Bouby N, Balkau B, Lantieri O, Alhenc-Gelas F, Marre M, Bankir L: Low water intake and risk for new-onset hyperglycemia. Diabetes Care 2011;34:25512554.

32 Sontrop JM, Dixon SN, Garg AX, BuendiaJimenez I, Dohein O, Huang SH, Clark WF: Association between water intake, chronic kidney disease, and cardiovascular disease: a cross-sectional analysis of NHANES data. Am J Nephrol 2013;37:434-442.

33 Clark WF, Sontrop JM, Macnab JJ, Suri RS, Moist L, Salvadori M, Garg AX: Urine volume and change in estimated GFR in a community-based cohort study. Clin J Am Soc Nephrol 2011;6:2634-2641.

34 Enhorning S, Bankir L, Bouby N, Struck J, Hedblad B, Persson M, Morgenthaler NG, Nilsson PM, Melander O: Copeptin, a marker of vasopressin, in abdominal obesity, diabetes and microalbuminuria: the prospective Malmo Diet and Cancer Study cardiovascular cohort. Int J Obes (Lond) 2013;37:598603.

35 Enhorning S, Hedblad B, Nilsson PM, Engstrom G, Melander O: Copeptin is an independent predictor of diabetic heart disease and death. Am Heart J 2015;169:549-556.e1.

36 Enhorning S, Struck J, Wirfalt E, Hedblad B, Morgenthaler NG, Melander O: Plasma copeptin, a unifying factor behind the metabolic syndrome. J Clin Endocrinol Metab 2011;96: E1065-E1072.

37 Enhorning S, Wang TJ, Nilsson PM, Almgren P, Hedblad B, Berglund G, Struck J, Morgenthaler NG, Bergmann A, Lindholm E, Groop L, Lyssenko V, Orho-Melander M, NewtonCheh C, Melander O: Plasma copeptin and the risk of diabetes mellitus. Circulation 2010; 121:2102-2108.

38 Carroll HA, Betts JA, Johnson L: An investigation into the relationship between plain water intake and glycated $\mathrm{Hb}$ (HbAlc): a sexstratified, cross-sectional analysis of the UK National Diet and Nutrition Survey (20082012). Br J Nutr 2016:1-11.

39 Borghi L, Meschi T, Amato F, Briganti A, Novarini A, Giannini A: Urinary volume, water and recurrences in idiopathic calcium nephrolithiasis: a 5-year randomized prospective study. J Urol 1996;155:839-843.

40 Guelinckx I, Vecchio M, Perrier ET, Lemetais G: Fluid intake and vasopressin: connecting the dots. Ann Nutr Metab 2016;68(suppl 2): 6-11.
41 Strippoli GF, Craig JC, Rochtchina E, Flood VM, Wang JJ, Mitchell P: Fluid and nutrient intake and risk of chronic kidney disease. Nephrology (Carlton) 2011;16:326-334.

42 Palmer SC, Wong G, Iff S, Yang J, Jayaswal V, Craig JC, Rochtchina E, Mitchell P, Wang JJ, Strippoli GF: Fluid intake and all-cause mortality, cardiovascular mortality and kidney function: a population-based longitudinal cohort study. Nephrol Dial Transplant 2014;29: 1377-1384.

43 Curhan GC, Willett WC, Knight EL, Stampfer MJ: Dietary factors and the risk of incident kidney stones in younger women: Nurses' Health Study II. Arch Intern Med 2004; 164: 885-891.

44 de La Gueronniere V, Le Bellego L, Jimenez IB, Dohein O, Tack I, Daudon M: Increasing water intake by 2 liters reduces crystallization risk indexes in healthy subjects. Arch Ital Urol Androl 2011;83:43-50.

45 Türk C, Knoll T, Petrik A, Sarica K, Skolarikos A, Straub M, Seitz C: Guidelines on Urolithiasis. European Association of Urology, 2015. http://uroweb.org/wp-content/uploads/22Urolithiasis_LR_full.pdf.

46 Terzic $\mathrm{D}$, Johansson-Fällgren $\mathrm{O}$, Ragnarsson JP, Goetze O, Hammarsten O: Evaluation of a sensitive copeptin assay for clinical measurement. Open Clin Chem J 2012;5:21-26.

47 Tasevska I, Enhörning S, Christensson A, Persson M, Nilsson PM, Melander O: Increased levels of copeptin, a surrogate marker of arginine vasopressin, are associated with an increased risk of chronic kidney disease in a general population. Am J Nephrol 2016;44: 22-28.

48 Roussel R, Matallah N, Bouby N, El Boustany R, Potier L, Fumeron F, Mohammedi K, Balkau B, Marre M, Bankir L, Velho G: Plasma copeptin and decline in renal function in a cohort from the community: The prospective D.E.S.I.R. study. Am J Nephrol 2015;42:107-114.

49 Munoz CX, Johnson EC, Demartini JK, Huggins RA, McKenzie AL, Casa DJ, Maresh CM, Armstrong LE: Assessment of hydration biomarkers including salivary osmolality during passive and active dehydration. Eur J Clin Nutr 2013;67:1257-1263.

50 Eckford SD, Keane DP, Lamond E, Jackson SR, Abrams P: Hydration monitoring in the prevention of recurrent idiopathic urinary tract infections in pre-menopausal women. Br J Urol 1995;76:90-93.

51 Armstrong LE, Maresh CM, Castellani JW, Bergeron MF, Kenefick RW, LaGasse KE, Riebe D: Urinary indices of hydration status. Int J Sport Nutr 1994;4:265-279.

52 Armstrong LE, Soto JA, Hacker FT Jr, Casa DJ, Kavouras SA, Maresh CM: Urinary indices during dehydration, exercise, and rehydration. Int J Sport Nutr 1998;8:345-355.

53 Perrier ET, Bottin JH, Vecchio M, Lemetais G: Criterion values for urine-specific gravity and urine color representing adequate water intake in healthy adults. Eur J Clin Nutr 2017, Epub ahead of print. 
54 Kavouras SA, Johnson EC, Bougatsas D, Arnaoutis G, Panagiotakos DB, Perrier E, Klein A: Validation of a urine color scale for assessment of urine osmolality in healthy children. Eur J Nutr 2016;55:907-915.

55 McKenzie AL, Munoz CX, Ellis LA, Perrier ET, Guelinckx I, Klein A, Kavouras SA, Armstrong LE: Urine color as an indicator of urine concentration in pregnant and lactating women. Eur J Nutr 2017;56:355-362.

56 Elmadfa I, Meyer AL: Patterns of drinking and eating across the European Union: implications for hydration status. Nutr Rev 2015; 73(suppl 2):141-147.

57 Rittig S, Knudsen UB, Norgaard JP, Pedersen EB, Djurhuus JC: Abnormal diurnal rhythm of plasma vasopressin and urinary output in patients with enuresis. Am J Physiol 1989; 256(4 pt 2):F664-F671.

58 Iglesia I, Santaliestra-Pasias AM, Bel-Serrat S, Sadalla-Collese T, Miguel-Berges ML, Moreno LA: Fluid consumption, total water intake and first morning urine osmolality in Spanish adolescents from Zaragoza: data from the HELENA study. Eur J Clin Nutr 2016;70:541547.

59 Bottin JH, Lemetais G, Poupin M, Jimenez L, Perrier ET: Equivalence of afternoon spot and 24-h urinary hydration biomarkers in freeliving healthy adults. Eur J Clin Nutr 2016;70: 904-907.
60 Bar-David Y, Urkin J, Kozminsky E: The effect of voluntary dehydration on cognitive functions of elementary school children. Acta Paediatr 2005;94:1667-1673.

61 Bar-David Y, Urkin J, Landau D, Bar-David Z, Pilpel D: Voluntary dehydration among elementary school children residing in a hot arid environment. J Hum Nutr Diet 2009;22: 455-460.

62 Fadda R, Rapinett G, Grathwohl D, Parisi M, Fanari R, Calo CM, Schmitt J: Effects of drinking supplementary water at school on cognitive performance in children. Appetite 2012; 59:730-737.

63 Stookey JD, Brass B, Holliday A, Arieff A What is the cell hydration status of healthy children in the USA? Preliminary data on urine osmolality and water intake. Public Health Nutr 2012;15:2148-2156.

64 Chang T, Ravi N, Plegue MA, Sonneville KR, Davis MM: Inadequate hydration, BMI, and obesity among US adults: NHANES 20092012. Ann Fam Med 2016;14:320-324.

65 Armstrong LE, Johnson EC, McKenzie AL, Munoz CX: An empirical method to determine inadequacy of dietary water. Nutrition 2016;32:79-82.

66 Katz AI, Massry S, Agmon J, Toor M: Concentration and dilution of urine in permanent inhabitants of hot regions. Isr J Med Sci 1965;1:968-978.
67 Manz F, Wentz A: 24-h hydration status: parameters, epidemiology and recommendations. Eur J Clin Nutr 2003;57(suppl 2):S10 S18.

68 Perrier ET, Buendia-Jimenez I, Vecchio M Armstrong LE, Tack I, Klein A: Twenty-fourhour urine osmolality as a physiological index of adequate water intake. Dis Markers 2015; 2015:231063.

69 Cheuvront SN, Kenefick RW: Am I drinking enough? Yes, no, and maybe. J Am Coll Nutr 2016;35:185-192.

70 Roussel R, El Boustany R, Bouby N, Potier L, Fumeron F, Mohammedi K, Balkau B, Tichet J, Bankir L, Marre M, Velho G: Plasma copeptin, AVP gene variants, and incidence of type 2 diabetes in a cohort from the community. J Clin Endocrinol Metab 2016;101:24322439.

71 Santillan MK, Santillan DA, Scroggins SM, Min JY, Sandgren JA, Pearson NA, Leslie KK, Hunter SK, Zamba GK, Gibson-Corley KN, Grobe JL: Vasopressin in preeclampsia: a novel very early human pregnancy biomarker and clinically relevant mouse model. Hypertension 2014;64:852-859.

72 Yeung EH, Liu A, Mills JL, Zhang C, Mannisto T, Lu Z, Tsai MY, Mendola P: Increased levels of copeptin before clinical diagnosis of preeclampsia. Hypertension 2014;64:13621367. 